\title{
High Surface Area Graphene Foams by Chemical Vapor Deposition
}

\author{
Simon Drieschner ${ }^{1}$, Michael Weber ${ }^{1}$, Jörg Wohlketzetter ${ }^{1}$, \\ Josua Vieten $^{1}$, Evangelos Makrygiannis ${ }^{1}$, Benno M. Blaschke ${ }^{1}$, \\ Vittorio Morandi ${ }^{2}$, Luigi Colombo ${ }^{3}$, Francesco Bonaccorso ${ }^{4}$, \\ and Jose A. Garrido ${ }^{5,6}$
}

${ }^{1}$ Walter Schottky Institut und Physik-Department, Technische Universität München, Am Coulombwall 4, 85748 Garching, Germany

${ }^{2}$ CNR-IMM via Gobetti 101, 40129 Bologna, Italy

${ }^{3}$ Analog Technology Development, Texas Instruments 13121 TI Blvd MS-367, Dallas, TX 75243, USA

${ }^{4}$ Istituto Italiano di Tecnologia, Graphene Labs Via Morego 30, 16163 Genova, Italy

${ }^{5}$ ICN2 - Catalan Institute of Nanoscience and Nanotechnology, Barcelona Institute of Science and Technology and CSIC, Campus UAB, 08193 Bellaterra, Spain

${ }^{6}$ ICREA, Institució Catalana de Recerca i Estudis Avançats, 08070 Barcelona, Spain

E-mail: joseantonio.garrido@icn2.cat

\begin{abstract}
.
Three-dimensional (3D) graphene-based structures combine the unique physical properties of graphene with the opportunity to get high electrochemically available surface area per unit of geometric surface area. Several preparation techniques have been reported to fabricate 3D graphene-based macroscopic structures for energy storage applications such as supercapacitors. Although reaserch has been focused so far on achieving either high specific capacitance or high volumetric capacitance, much less attention has been dedicated to obtain high specific and high volumetric capacitance simultaneously. Here, we present a facile technique to fabricate graphene foams (GF) of high crystal quality with tunable pore size grown by chemical vapor deposition. We exploited porous sacrificial templates prepared by sintering nickel and copper metal powders. Tuning the particle size of the metal powders and the growth temperature allow fine control of the resulting pore size of the 3D graphene-based structures smaller than $1 \mu \mathrm{m}$. The as-produced 3D graphene structures provide a high volumetric electric double layer capacitance $\left(165 \mathrm{mF} \mathrm{cm}^{-3}\right)$. High specific capacitance $\left(100 \mathrm{~F} \mathrm{~g}^{-1}\right)$ is obtained by lowering the number of layers down to single layer graphene. Furthermore, the small pore size increases the stability of these GFs in contrast to the ones that have been grown so far on commercial metal foams. Electrodes based on the as-prepared GFs can be a boost for the development of supercapacitors, where both low volume and mass are required.
\end{abstract}

Keywords: graphene foams, CVD, EDLC, powders 


\section{Introduction}

Graphene foam (GF)[1], a three-dimensional (3D) interconnected network consisting of graphene sheets[2], is one of the latest developed form of graphene-based materials[1, 3, 4]. These structures are of great scientific interest since they take advantage of graphene's unique physical properties such as high electrical[5] and thermal conductivity[6], biocompatibility[7], high mechanical[8] and chemical[9] stability while at the same time they can provide a very high electrochemically available surface (EASA) per unit of geometric area. These properties make GFs very promising for electric double layer capacitors (EDLCs), also known as supercapacitors[10, 11] as well as for sensing applications[12]. In EDLCs, typically based on two symmetric electrodes impregnated with electrolytes[13], the capacitance is directly proportional to the area of the electric double layer formed at the interface between the electrodes and the electrolyte; thus, large areas are necessary to obtain high capacitance values[11]. However, while a sheet of graphene has a theoretical specific surface area (SSA) of $2630 \mathrm{~m}^{2} \mathrm{~g}^{-1}$ [14], which is significantly larger than the one reported to date for carbon nanotubes (CNTs), $\approx 100-1000 \mathrm{~m}^{2} \mathrm{~g}^{-1}[14]$, or carbon black $\left(<900 \mathrm{~m}^{2} \mathrm{~g}^{-1}\right)[15]$, and similar to activated carbon[16], applications of these materials as supercapacitor electrodes do not only require high SSA but also a high ratio between the EASA and the geometric area[17] of the device, i.e. the so-called roughness factor[18]. Electrodes with large roughness factors are preferred when designing EDLCs with high capacitance[18, 19]. However, considering a flat electrode based on a single layer graphene (SLG) film, the roughness factor equals 1. Therefore, in order to realize electrodes based on SLG exhibiting high EASA, a method for the formation of 3D structures needs to be developed. Many methods have been used to synthesize graphene-based 3D structures. For example, chemical reduction of graphene oxide $(\mathrm{GO})[20,21]$ has been widely used to create graphene-based 3D structures with SSA values[22, 23] up to $3100 \mathrm{~m}^{2} \mathrm{~g}^{-1}[16]$. More advanced preparation methods based on mixtures of GO with polymers have been shown to yield SSAs up to $3523 \mathrm{~m}^{2} \mathrm{~g}^{-1}[24]$. However, the final reduced graphene oxide (RGO) obtained by these methods typically suffer from a high defect density[21] and possible poor connections of the graphene sheets in $3 \mathrm{D}$ structures. This can drastically reduce the electrical conductivity of the GFs based on this material[25], which is detrimental to the performance of RGO-based electrodes for EDLCs[11]. In contrast, chemical vapor deposition (CVD) can provide large area graphene films of high crystalline

quality[26]. Following this idea, it has been previously demonstrated[1] that using 3D microporous metal substrates, also referred to as metal foams, an interconnected graphene network can be created after wet chemical etching of the metal template used for growing graphene[1]. Nevertheless, since the graphene film conformably covers the metal template, the resulting morphology and pore size distribution of the as-prepared GF is strongly determined by the structure of the template itself[1]. For example, commercially available copper $(\mathrm{Cu})$ and nickel $(\mathrm{Ni})$ foams, previously used to demonstrate the growth of high quality SLG and few layer graphene (FLG), 
respectively[1], have a typical minimum pore size of $\approx 200 \mu \mathrm{m}[1]$. As a result, the volumetric capacitance, i.e. the capacitance per unit volume, of GFs based on these commercial substrates is limited by the large void volume in the rather large pores (i.e. $200-400 \mu \mathrm{m}$ ), which does not contribute to the EDLC[27]. Moreover, since the pores in the foam have to be filled by an electrolyte, which has the largest contribution to the total mass of the EDLC[27], the specific capacitance of the EDLC, i.e. the capacitance per unit mass, is severely limited[27]. It is experimentally observed that after the etching of the metal template and during the drying step, the SLG-based 3D structure prepared using commercial $\mathrm{Cu}$ foams cannot remain free-standing due to the large voids $(\approx 200-400 \mu \mathrm{m})$ and eventually collapses, thus limiting the mechanical stability of these foams. Therefore, GFs with small pore size (significantly smaller than the values currently obtained with commercial metal foams, i.e. $\ll 200 \mu \mathrm{m}$ ) like the graphene-like 3D carbon obtained by the use of zeolite templates[28] are key to achieve both high specific and volumetric capacitance, as well as enhanced mechanical stability. The fabrication of carbon foams starting from Ni nanowires[29], $\mathrm{Ni}$ powder[30], Ni particle and carbohydrate mixtures[31], Ni chloride powder[32], or $\mathrm{Ni}$ manganese alloys[33] have been recently reported. However, these studies did not report on the control of the resulting pore dimensions. The pore sizes in graphene nano-networks fabricated using poly(vinyl alcohol)/iron infiltrated into 3D-assembledcolloidal silicas might be tunable by the size of the silica spheres[34]. However, this and all the other reported materials[29, 31, 32, 33] did not exhibit single-layer graphene characteristics. Other reports on materials exhibiting high capacitance values such as doped carbon microspheres[35] or MXenes[36] focus on either specific or volumetric capacitance without controlling the pore size. Furthermore, they use components such as nitrogen[35] and fluorine[35, 36] which are electrochemically active and thus show a high pseudocapacitive contribution[37].

In this work, we present a method for synthesizing 3D SLG- and FLG-based GFs by CVD on metal foams with controllable pore size providing both high specific and volumetric electric double layer capacitance when the as-produced foams are used as electrodes in aqueous electrolytes. We accomplished this by preparing the metal templates by sintering metal powders at high temperatures $\left(600-1100^{\circ} \mathrm{C}\right)$ rather than using commercially available foams. The density and dimension of the pores were tuned on-demand by optimizing the growth temperature and the catalyst particle size. Both $\mathrm{Ni}$ and $\mathrm{Cu}$ powders were used to create metal templates with a pore size down to $1 \mu \mathrm{m}$. In particular, in the case of $\mathrm{Cu}$ particles, pores smaller than $1 \mu \mathrm{m}$ were obtained at a growth temperatures as high as $1000^{\circ} \mathrm{C}$. Following the fabrication of these $3 \mathrm{D}$ metal structures, SLG and FLG were grown via CVD[38] using methane[39] as the carbon precursor, obtaining an interconnected and free-standing GF after wet etching of the metal template. In contrast to GFs grown on commercial metal foams[1], the lower pore size of both $\mathrm{Ni}$ - and $\mathrm{Cu}$-grown $\mathrm{GFs}$ enabled the formation of stable structures without any supporting polymer[1] even in the case of SLG films. In addition, the control over the pore size allows a $10^{4}$-fold increase in terms of surface area, which together with the 
stability of the free-standing foams represents a significant improvement over the state of the art GFs[39].

\section{Results and discussion}

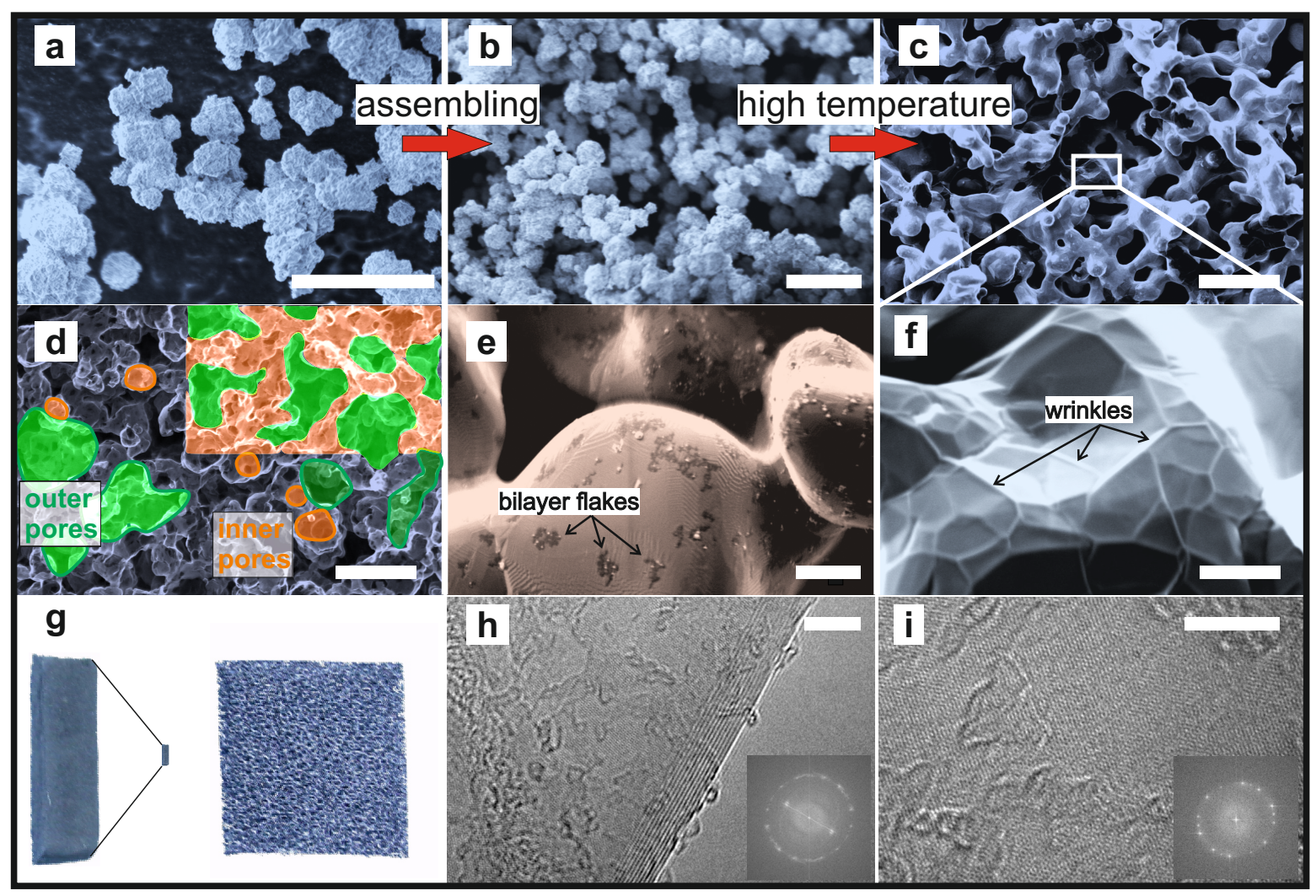

Figure 1. Principle of the fabrication of GF illustrated by scanning electron microscope (SEM) images. (a) Metal particles, in this case $\mathrm{Ni}$, are assembled in a vessel to get (b) a $3 \mathrm{D}$ structure. (c) At high temperatures $\left(\geq 600^{\circ} \mathrm{C}\right)$ interconnected metal foams are created; this process is implemented in the CVD annealing step. The scale bars are $20 \mu \mathrm{m}$. (d) Metal foam showing outer and inner pores. The scale bar is $10 \mu \mathrm{m}$. SEM images of graphene films showing (e) characteristic bilayer flakes on $\mathrm{Cu}$ and (f) wrinkles on Ni. The scale bars are (e) $5 \mu \mathrm{m}$ and (f) $1 \mu \mathrm{m}$. (g) Enlargement (left) of our $\mathrm{Ni}$ foam (center) compared to a commercial Ni foam (right) revealing the differences in volume for the same surface area. TEM characterization. (h) HRTEM of a folded edge. In the inset on the lower right corner, the FFT of the image shows the spots corresponding to the $0.335 \mathrm{~nm}$ (inner) and the $0.213 \mathrm{~nm}$ (outer) reflections. (i) HRTEM image of the Ni-grown GF, with the corresponding FFT in the inset. The scale bars are $5 \mathrm{~nm}$.

The principle of our preparation technique is illustrated in Fig. 1a-c. $\mathrm{Ni}$ or $\mathrm{Cu}$ powder (Fig. 1a) is loaded in a combustion vessel creating a homogeneously distributed 3D structure (Fig. 1b). In contrast to the fabrication of commercial metal foams, which are typically prepared using compressed powders with particles of hundreds of micrometers in size and employing metal hydrides as blowing agents[40], $\mathrm{Ni}$ and $\mathrm{Cu}$ 
powder of small particle size (average particle size smaller than $10 \mu \mathrm{m}$, see Fig. S2) were used without hydrides or compression. In the case of structures prepared using $\mathrm{Cu}$ particles in the sub-micrometer range $(\leq 1 \mu \mathrm{m}), \mathrm{MgCO}_{3}$ was used as a blowing agent to enable the release of $\mathrm{CO}_{2}$ at higher temperatures[41] $\left(>800^{\circ} \mathrm{C}\right)$, thus preventing the agglomeration of the metal particles. After filling the vessel, the particles are heated in a low pressure atmosphere $\left(50\right.$ mbar) to a temperature of at least $600{ }^{\circ} \mathrm{C}$ in the case of $\mathrm{Ni}$ and $800^{\circ} \mathrm{C}$ in the case of $\mathrm{Cu}$. At these temperatures, atoms at the touching points of the metal particles start to migrate without reaching the liquid state forming a bond between the metal particles themselves[42] and thus yielding an interconnected highly porous network (Fig. 1c). Annealing is carried out under flowing hydrogen and argon to reduce contamination[43] and remove metal oxides from the surface of the metal[44]. After introducing methane at $T \geq 600^{\circ} \mathrm{C}$ and at a $P \approx 50 \mathrm{mbar}$ in the chamber, $\mathrm{CH}_{4}$ molecules dehydrogenate[43] on the metal surface leading to graphene nucleation on the metal surface, e.g. for the case of $\mathrm{Cu}[43]$. The graphene growth process $[38,44,45,46]$ strongly depends on the metal catalyst[36]. For example, in the case of $\mathrm{Ni}[47]$, the carbon atoms diffuse into the bulk of the metal due to the high carbon solubility $(\approx 0.1 \mathrm{~g} / 100 \mathrm{~g})[48]$ and graphene is formed both by the isothermal growth on the surface[49] and carbon precipitation from the bulk upon cooling[50, 51]. The shortcoming of graphene growth on $\mathrm{Ni}$ is the poor control on the number of layers, which is strongly influenced by the thickness of the $\mathrm{Ni}$, temperature and time of exposure to the hydrocarbon, and the cooling rate[52] $\left(\approx 10\right.$ to $40{ }^{\circ} \mathrm{C}$ per minute in the present work), not yielding uniform SLG, but rather FLG[51]. It would be extremely difficult to control all of these parameters to promote the growth of graphene. In the case of $\mathrm{Cu}$, the process is self-limited, i.e. growth mostly ceases as soon as the $\mathrm{Cu}$ surface is fully covered with graphene principally because of the extremely low C solubility[46] and high $\mathrm{C}$ diffusivity in $\mathrm{Cu}[53]$, although a small percentage of the surface can be covered with few-layer graphene arising from the small amount of $\mathrm{C}$ dissolved in the $\mathrm{Cu}[53]$ and then precipitated to the $\mathrm{Cu}$ surface upon cooling.

Large area graphene growth is enabled principally by the low $\mathrm{C}$ solubility in $\mathrm{Cu}$ (7.4 ppm at $1020^{\circ} \mathrm{C}$ )[54], and the mild catalytic activity of the $\mathrm{Cu}$ metal[46]. Due to the self-limiting nature of the process[46] and the preferential SLG growth on $\mathrm{Cu}$, the resulting GF has very low mass. Small bilayer flakes and wrinkles created by different thermal expansion coefficients[55] are also observed in the films grown on $\mathrm{Cu}$ and $\mathrm{Ni}$, respectively (Fig. 1e and f). After graphene growth, the metal template (either Ni or $\mathrm{Cu}$ ) is dissolved in a hot $\mathrm{FeCl}_{3} / \mathrm{HCl}$ etching solution followed by residue removal in two baths of $\mathrm{HCl}$ and deionized water[1]. The samples are then freeze-dried (see Methods for details) to prevent the collapse of the GFs by the capillary force of water in the micrometer size pores. Finally, free-standing, homogeneous, and stable GFs with pore sizes in the micrometer range are obtained which are discussed in the following. Compared to GFs grown on commercial metal foams using the same conditions (see Fig. $1 \mathrm{~g}$ (right)), the GF prepared using our foams exhibit a notably more compact and dense structure (see Fig. $1 \mathrm{~g}$ (left)) resulting in a higher EASA per volume. For 
instance, whereas the GFs shown in Fig. 1g (left metal foam is prepared using our foam and the right image corresponds to a commercial $\mathrm{Ni}$ foam) have the same EASA (derived from cyclic voltammetry, see Methods section), the volume of the commercial foam is $10^{3}$ times larger than our $\mathrm{Ni}$ foam when using the same growth parameters for both GFs. Figs. 1h-i show the transmission electron microscopy (TEM) images of the $\mathrm{GF}$ grown on Ni. Further technical details and results for GFs grown on $\mathrm{Ni}$ and $\mathrm{Cu}$ templates are reported in the Methods section and in the Supporting Information (S.I.). High resolution TEM (HRTEM) analysis performed on the edges of the deposited flakes, counting the number of the visible (002) lattice fringes, allows for a careful determination of the local number of graphene layers, as shown in Fig. 1h, where a multi-layer portion of the graphene film folded over itself is shown. The fast Fourier transform (FFT) in the inset clearly shows the inner spots at $0.335 \mathrm{~nm}$, corresponding to (002) fringes, and the hexagonal spots at $0.213 \mathrm{~nm}$, typical of the graphene lattice. The HRTEM image shown in Fig. 1i and the corresponding FFT in the inset definitely confirm the crystallinity of the as-grown GFs.

The number of layers and the structural quality of the GFs are also investigated by Raman spectroscopy[56, 57]. Fig. 2a plots a typical Raman spectrum of the GFs grown on $\mathrm{Cu}$ at $900{ }^{\circ} \mathrm{C}$ and $\mathrm{Ni}$ at $600{ }^{\circ} \mathrm{C}$ for excitation wavelength of $532 \mathrm{~nm}$. Statistical analysis of the micro-Raman spectra (see S.I for more details) shows that the position of the $2 \mathrm{D}$ peak $(\operatorname{Pos}(2 \mathrm{D}))$, see Fig. $2 \mathrm{~b}$, is at $\approx 2695 \mathrm{~cm}^{-1}$ and $2699 \mathrm{~cm}^{-1}$ for $\mathrm{GFs}(\mathrm{Cu})$ and $\operatorname{GFs}(\mathrm{Ni})$, respectively, while the $\mathrm{FWHM}(2 \mathrm{D})$ are peaked at $\approx 61 \mathrm{~cm}^{-1}$ and $80 \mathrm{~cm}^{-1}$ for $\mathrm{Cu}$ and Ni-grown GFs, respectively. These values are consistent with the $\mathrm{Cu}$ grown GFs being mostly SLG while the Ni-grown GFs consist of FLG. The Raman spectrum also shows (Fig. $2 \mathrm{a}$ ) an average intensity ratio $\mathrm{I}(\mathrm{D}) / \mathrm{I}(\mathrm{G}) \approx 0.16$ and $\approx 0.25$ for $\mathrm{Cu}$ - and Ni-grown GFs, respectively (see S.I. for more details on statistical analysis). Raman maps recorded in a confocal 3D configuration (see Methods for details), were used to visualize the 3D structure of the GFs. Measurements at different focal planes are shown in the S.I., providing a 3D reconstruction of the GF which helps reveal its porous structure. Fig. 2c and d depict the FWHM(2D) at constant focal plane position of the GFs grown on $\mathrm{Ni}$ and $\mathrm{Cu}$, respectively. The average $\mathrm{FWHM}(2 \mathrm{D})$ is $\left(90.9 \pm 11.1 \mathrm{~cm}^{-1}\right)$ and $\left(70.0 \pm 9.8 \mathrm{~cm}^{-1}\right)$ indicating the presence of FLG and SLG on the Ni-grown and $\mathrm{Cu}$-grown $\mathrm{GFs}$, respectively. The temperature dependence of the $2 \mathrm{D}$ to $\mathrm{G}$ intensity ratio $(\mathrm{I}(2 \mathrm{D}) / \mathrm{I}(\mathrm{G})$ ) measured on $\mathrm{Ni}$ - and $\mathrm{Cu}$-grown GFs extracted from Raman maps is shown in Fig. 2e and $f$, respectively. The $\mathrm{I}(2 \mathrm{D}) / \mathrm{I}(\mathrm{G})$ ratio also helps to get information about the number of layers, although it might be influenced by doping and strain[58]. However, these effects are assumed to be small since the GFs are freestanding, i.e. strong doping induced by polymers typically used to support the GF during the transfer and the impact of a substrate can be excluded. The $\mathrm{I}(2 \mathrm{D}) / \mathrm{I}(\mathrm{G})$ is $0.81 \pm 0.48$ at $600{ }^{\circ} \mathrm{C}$ for $\mathrm{GFs}(\mathrm{Ni})$ and $2.29 \pm 1.57$ at $800^{\circ} \mathrm{C}$ for $\mathrm{GFs}(\mathrm{Cu})$ suggesting the presence of FLGs and SLGs, respectively. Increasing the growth temperature leads to slightly lower $\mathrm{I}(2 \mathrm{D}) / \mathrm{I}(\mathrm{G})$ values especially for $\mathrm{Cu}$-grown $\mathrm{GFs}\left(0.69 \pm 0.41\right.$ at $\left.1000{ }^{\circ} \mathrm{C}\right)$ with respect to the one obtained for lower growth temperature $\left(600^{\circ} \mathrm{C}\right)$. The obtained 
$\mathrm{I}(2 \mathrm{D}) / \mathrm{I}(\mathrm{G})$ values coupled with a widening of the $\mathrm{FWHM}(2 \mathrm{D})$ indicates the presence of multilayer graphene in the GFs grown at higher temperatures, i.e. $1000^{\circ} \mathrm{C}$. At $600{ }^{\circ} \mathrm{C}$, the average intensity ratio $\mathrm{I}(\mathrm{D}) / \mathrm{I}(\mathrm{G})$ is $0.22 \pm 0.01$ suggesting the presence of defects on the Ni-grown GFs. This value decreases down to $\approx 0.03$ for GFs grown at higher temperatures (i.e. $1100^{\circ} \mathrm{C}$ ), as depicted in Fig. 2e. A higher growth temperature leads to a higher decomposition rate of methane[43] and given the higher solubility of $\mathrm{C}$ in $\mathrm{Ni}$ it results in a higher number of graphene layers and a lower defect density, as reported by ref. [51]. The values of $\mathrm{I}(\mathrm{D}) / \mathrm{I}(\mathrm{G})$ for the $\mathrm{GFs}$ grown on $\mathrm{Cu}$ show a similar trend as the ones reported for Ni-grown GFs. In particular, the $\mathrm{I}(\mathrm{D}) / \mathrm{I}(\mathrm{G})$ decreased from $0.87 \pm 0.27\left(800^{\circ} \mathrm{C}\right)$ to $0.15 \pm 0.05\left(1000^{\circ} \mathrm{C}\right)$ (see Fig. 2e and f.) This can be understood by considering that high growth temperatures $\left(1000^{\circ} \mathrm{C}\right)$ typically yield larger graphene
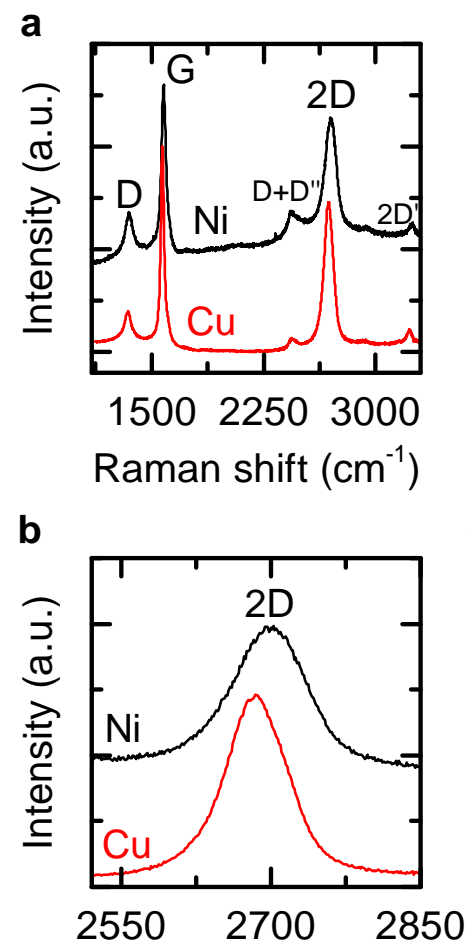

Raman shift $\left(\mathrm{cm}^{-1}\right)$
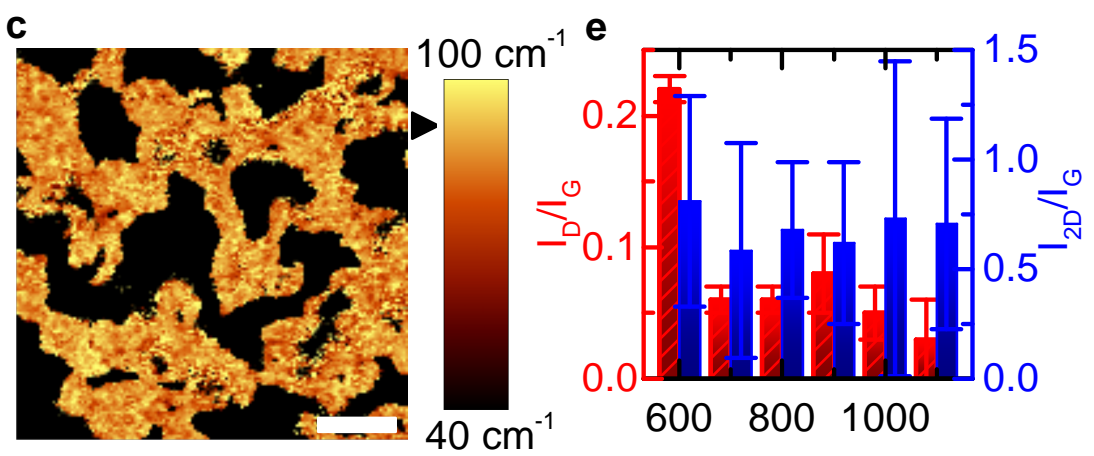

FWHM(2D)

Growth temperature $\left({ }^{\circ} \mathrm{C}\right)$
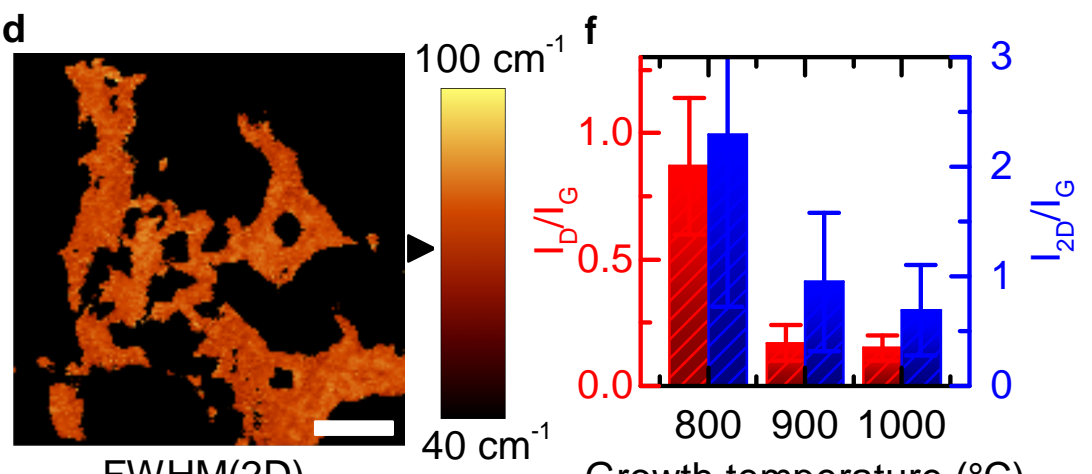

Growth temperature $\left({ }^{\circ} \mathrm{C}\right)$

Figure 2. Raman characterization of as-grown GFs on $\mathrm{Ni}$ and $\mathrm{Cu}$ templates. (a) Raman spectra of $\mathrm{GF}$ grown on $\mathrm{Cu}$ (red curve) at $900^{\circ} \mathrm{C}$ and $\mathrm{Ni}$ (black curve) at $600^{\circ} \mathrm{C}$. (b) Close-up of the 2D Raman peak for GF grown on $\mathrm{Cu}$ (red curve) and $\mathrm{Ni}$ (black curve). Confocal Raman maps showing the FWHM of the 2D peak for graphene films grown on (c) $\mathrm{Ni}$ and (d) $\mathrm{Cu}$ at $600^{\circ} \mathrm{C}$ and $900^{\circ} \mathrm{C}$, respectively. The arrows in the color scale point to the average values. The scale bars are $5 \mu \mathrm{m}$. Growth temperature dependence of the $\mathrm{I}(\mathrm{D}) / \mathrm{I}(\mathrm{G})$ (red columns) and $\mathrm{I}(2 \mathrm{D}) / \mathrm{I}(\mathrm{G})$ (blue columns) intensities ratio of graphene films grown on (e) $\mathrm{Ni}$ and (f) $\mathrm{Cu}$, respectively. The growths were carried out by using $\mathrm{Ni}$ (size A) and $\mathrm{Cu}$ (size A) powders. 
domain sizes in the lower micrometer range (1-5 $\mathrm{m}$, see Fig. S23) and therefore lower grain boundary densities[59] with respect to the growth at $800{ }^{\circ} \mathrm{C}$; as a result, the $\mathrm{D}$ peak intensity is reduced as the growth temperature is increased.

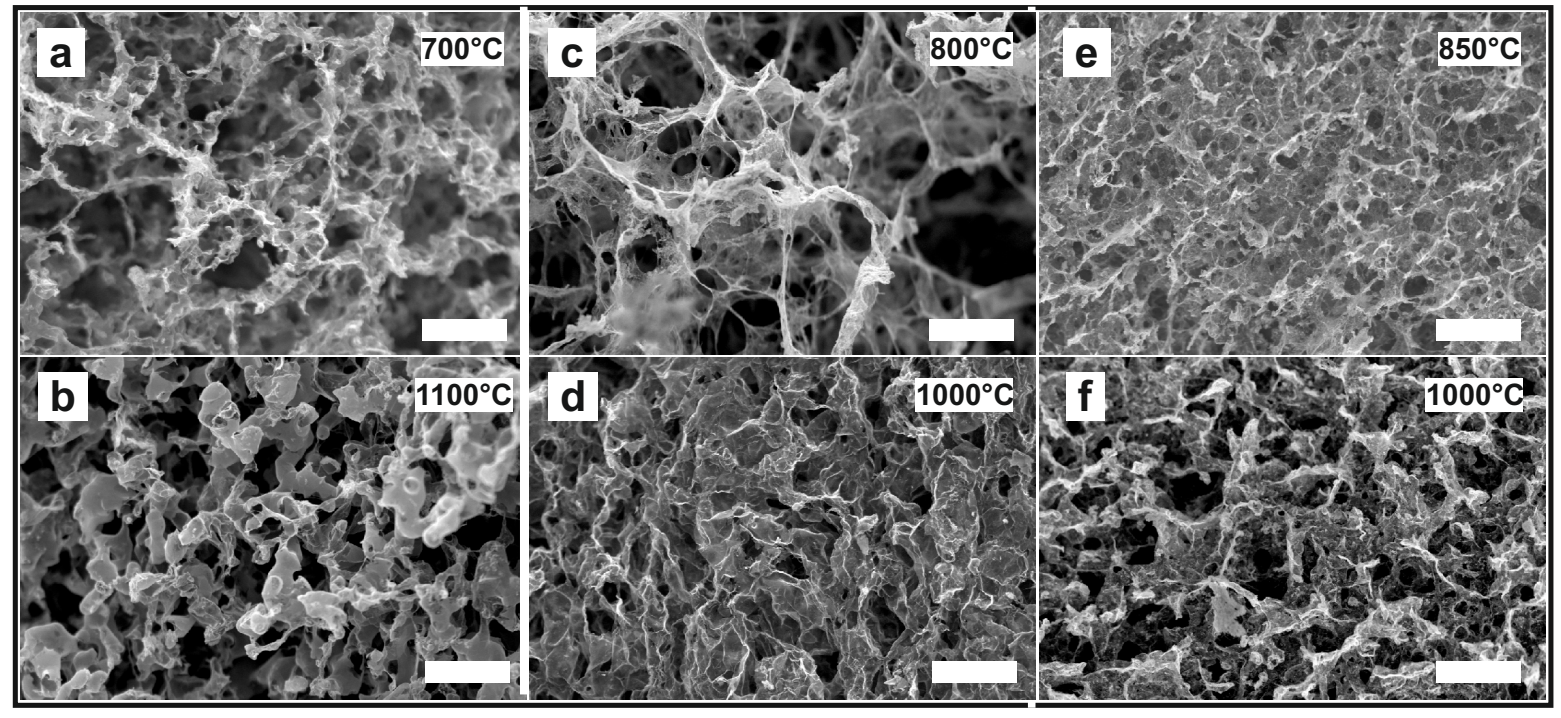

Figure 3. SEM characterization of as-grown GFs on $\mathrm{Ni}$ and $\mathrm{Cu}$ templates. SEM micrographs showing the morphology of the free-standing GFs grown on Ni (size B) (a)(b), $\mathrm{Cu}($ size A) (c)-(d), and $\mathrm{Cu}(\mathrm{Mg}) 1 \mu \mathrm{m}(\mathrm{e})-(\mathrm{f})$ templates at different temperatures. The maximum particle size of the used powder is $50 \mu \mathrm{m}$ for Ni (a)-(b) and $135 \mu \mathrm{m}$ for $\mathrm{Cu}$ templates (c)-(d). For the $\mathrm{Cu}$ template in (e)-(f), $\mathrm{Cu}$ (size B) powder mixed with $\mathrm{MgCO}_{3}$ was used. The scale bars are $20 \mu \mathrm{m}$.

The influence of the growth temperature on the pore size of the GFs prepared using $\mathrm{Ni}$ and $\mathrm{Cu}$ powders has been assessed by SEM. Fig. 3a and b correspond to Ni powders (size B) and Fig. 3c and d to $\mathrm{Cu}$ powders (size A), both with a particle size smaller than $50 \mu \mathrm{m}$ (average particle size below $10 \mu \mathrm{m}$ ). The GFs shown in Fig. 3e and f were grown on templates using a $1 \mu \mathrm{m} \mathrm{Cu}$ powder (size B) mixed with $\mathrm{MgCO}_{3}$ powder with a mass ratio of 9:1, which is referred to as $\mathrm{Cu}(\mathrm{Mg}) 1 \mu \mathrm{m}$ in the following (see Methods and S.I. for details). Additional SEM images of GFs prepared with Ni powders of different particle size are provided in the S.I.. In order to discuss the dependence of the EASA on the growth temperature, two different types of pores are distinguished. We define the so-called outer pore as the void space limited by arms of the metal template (see Fig. 1d); the so-called inner pore is defined as the void space created by the metal etching and therefore as the arm width of the metal arms (see Fig. 1d).

A quantitative analysis of these pores is depicted in Fig. 4a, where the arm width, which is associated with the diameter of the inner pores, and the average outer pore diameter is plotted versus the growth temperature for the Ni powder (size B). At low growth temperatures $\left(700^{\circ} \mathrm{C}\right.$ in the case of the $\mathrm{GF}$ shown in Fig. 3a), many outer pores larger than $20 \mu \mathrm{m}$ can be observed, and the average outer pore size is $24 \mu \mathrm{m}$ (see Fig. 4a). Increasing the growth temperature $\left(>700^{\circ} \mathrm{C}\right)$ leads to a reduction of 
the average outer pore size of the resulting GFs. In the case of $\mathrm{Ni}$, narrow outer pores with a diameter smaller than $20 \mu \mathrm{m}$ can be observed (Fig. 3b). Using this powder, the dimensions of the outer pores can be tuned between 12 and $30 \mu \mathrm{m}$. The diameter of the inner pores shows a different growth temperature dependence with respect to the one of the outer pores. For the Ni powder, the average arm width and therefore the dimension of the inner pores increases from $\approx 2 \mu \mathrm{m}$ at $600{ }^{\circ} \mathrm{C}$ to $\approx 5 \mu \mathrm{m}$
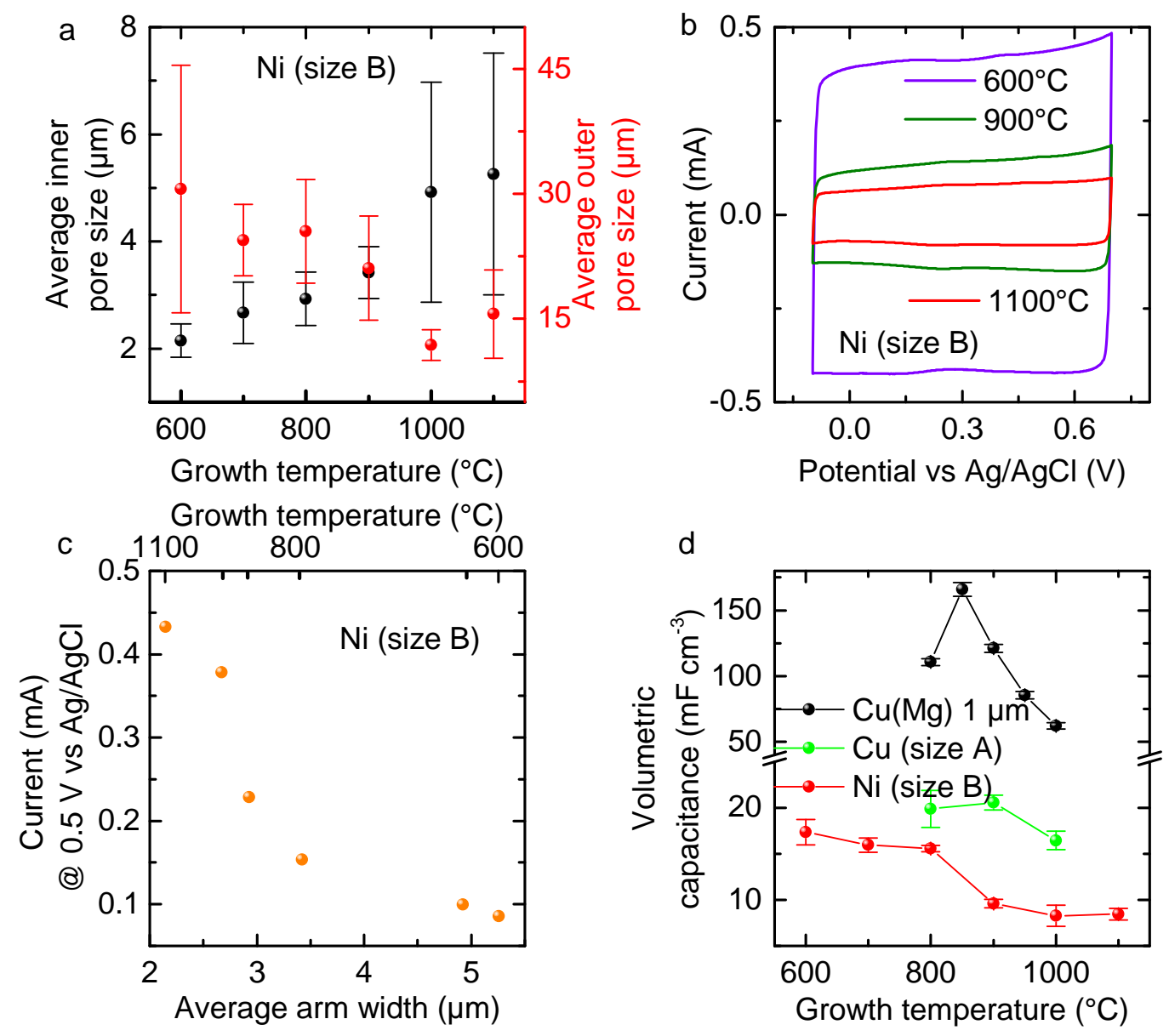

Figure 4. Growth temperature dependence of the foam structure. (a) Growth temperature dependence of the average measured inner pore size and the average outer pore size of GFs prepared using Ni templates (size B powder). (b) Cyclic voltammetry (CV) curves of GFs grown on Ni templates at three different temperatures, measured in a $3 \mathrm{M} \mathrm{KCl}$ electrolyte. The scan rate is $100 \mathrm{mV} \mathrm{s}^{-1}$. The current of the CV curves is not normalized by any mass or volume, since the same amount of metal particles $(443 \pm 13 \mathrm{mg})$ is used for every sample, i.e. the mass of the metal foam measured before the transfer (etching) is the same for every sample. (c) Influence of the growth temperature on the current at $0.5 \mathrm{~V}$ vs $\mathrm{Ag} / \mathrm{AgCl}$ extracted from the $\mathrm{CV}$ curves reported in (b). The growth temperature is shown on the top axis, whereas the bottom axis shows the calculated average arm width for each temperature. (d) Volumetric capacitance for some investigated GFs grown on metal particles calculated from charge-discharge measurements at $1 \mathrm{~mA} \mathrm{~cm}^{-3}$. 
at $1100{ }^{\circ} \mathrm{C}$ (see Fig. 4a). Graphene foams grown on $\mathrm{Cu}$ templates (Fig. 3c-f) show the same growth temperature dependence for both the inner and outer pores (see S.I. for quantitative analysis). Additionally, the number of graphene layers has an important effect on the resulting structure of GFs prepared on $\mathrm{Cu}$ templates, which we attempt to discuss in terms of the different mechanical properties of SLG and FLG. At low growth temperatures $\left(800^{\circ} \mathrm{C}\right.$ and $850{ }^{\circ} \mathrm{C}$, Fig. 3c and e, respectively), the flexibility of SLG[60] leads to a partial collapse of the inner pores, while at higher temperatures $\left(1000^{\circ} \mathrm{C}\right)$ the higher rigidity of FLG $[60]$ preserves the original structure of the $\mathrm{Cu}$ foam (see Fig. 3d and f). In order to assess the EASA of the GFs, cyclic voltammetry (CV) curves have been measured in a $3 \mathrm{M} \mathrm{KCl}$ aqueous electrolyte for different growth temperatures (Fig. 4b). The CV curves show, over the whole electrochemical potential window $(-0.1$ to $0.7 \mathrm{~V})$, a quasi-rectangular shape without any peaks resulting from redox reactions typical of faradaic processes[19], confirming an EDLC behavior. The current, and thus the capacitance, increase with decreasing GF growth temperature, which can be explained by the effect of the growth temperature on the structure of the resulting GFs. The average arm thickness for Ni foams (Fig. 4a) shows a decrease from $\approx 5 \mu \mathrm{m}$ to $\approx 2 \mu \mathrm{m}$ when decreasing the growth temperature from $1100{ }^{\circ} \mathrm{C}$ to $600{ }^{\circ} \mathrm{C}$. According to a simple qualitative model which assumes a cylindrical shape of the arms in the metal template (see S.I. for further discussion), this leads to an increase of the EASA which is proportional to the measured current. The pore size does not have an additional effect on the EASA, since the minimum pore size achieved in this work is $\approx 0.5 \mu \mathrm{m}$ (Fig. S16) and thus the majority of the pores are accessible to the ions in the electrolyte. The current plotted as a function of the average arm width for a Ni-grown foam (Fig. 4c) confirms the inverse relationship between EASA values and arm width. Fig. 4d presents the volumetric capacitance results of the GFs grown from $\mathrm{Ni}$ and $\mathrm{Cu}$ powders having different particle sizes. For all types of powder, the volumetric capacitance of the EDLC increases with decreasing growth temperature, which is the result of the gain in EASA discussed above. The drop of the volumetric capacitance of both $\mathrm{Cu}$ and $\mathrm{Ni}$ powders observed between $900^{\circ} \mathrm{C}$ and $800^{\circ} \mathrm{C}$ and $850^{\circ} \mathrm{C}$ and $800{ }^{\circ} \mathrm{C}$, respectively, can be explained by the collapse of the inner pores (compare Fig. 3c and e). The volumetric capacitance for the $\mathrm{Cu}(\mathrm{Mg}) 1 \mu \mathrm{m}$ powder is increased to $165 \mathrm{mF} \mathrm{cm}^{-3}$, while the highest specific capacitance is $100 \mathrm{~F} \mathrm{~g}^{-1}$. Considering the theoretical capacitance of SLG[61] yields an electrochemically active area of around $2500 \mathrm{~m}^{2} \mathrm{~g}^{-1}$ which is close to the theoretical limit of $2630 \mathrm{~m}^{2} \mathrm{~g}^{-1}$. Compared to GFs grown and transferred from commercially available Ni foams, GFs fabricated using our new method with a typical pore size of 0.5 to $30 \mu \mathrm{m}$ (see Fig. 4a and S15) exhibit a pore size that is up to three orders smaller than that of commercial foams (200 to $400 \mu \mathrm{m}$, see Fig. S1). Consequently, the volumetric capacitance of GFs grown on commercially available Ni scaffolds using the same growth parameters is only around $\left(5 \mu \mathrm{F} \mathrm{cm}^{-3}\right)$ and therefore almost five orders of magnitude smaller than the volumetric capacitance $\left(165 \mathrm{mF} \mathrm{cm}^{-3}\right)$ of $\mathrm{GFs}_{\text {grown on our }}$ customized $\mathrm{Cu}$ templates. For application in EDLCs, the most promising material is the GF grown using $\mathrm{Cu}(\mathrm{Mg}) 1 \mu \mathrm{m}$ powder grown at $850^{\circ} \mathrm{C}$ exhibiting the highest volumetric 
capacitance of $165 \mathrm{mF} \mathrm{cm}^{-3}$, the highest capacitance per area of $59 \mathrm{mF} \mathrm{cm}^{-2}$, and the highest specific capacitance of $100 \mathrm{~F} \mathrm{~g}^{-1}$. The corresponding energy and power density are $11.2 \mathrm{~W} \mathrm{~h} \mathrm{~kg}^{-1}$ and $10.1 \mathrm{~kW} \mathrm{~kg}^{-1}$, respectively.

The electrochemical characterization in $3 \mathrm{M} \mathrm{KCl}$ of a $\mathrm{GF}$ grown at $950^{\circ} \mathrm{C}$ using the $\mathrm{Cu}(\mathrm{Mg}) 1 \mu \mathrm{m}$ powder is depicted in Fig. 5 (see Figure S13 for a electrochemical characterization of foams prepared using Ni powders). The CV curves show no current peak that can be attributed to a Faradaic process, and remain quasi-rectangular for the entire range of scan rates (up to $300 \mathrm{mV} \mathrm{s}^{-1}$ ). In addition, electrochemical impedance spectroscopy measurements (see S.I. for further information) confirm the EDLC behavior of the GF, with a phase close to $-90^{\circ}$ at low frequencies as expected for an ideal capacitor[62]. The equivalent series resistance is $\approx 20 \Omega$, which can be mostly attributed to both the multilayer graphene sheet used as substrate to contact the foams and the resistance of the electrolyte (for further information see the Methods section and S.I.), causing small deviations from the quasi-rectangular
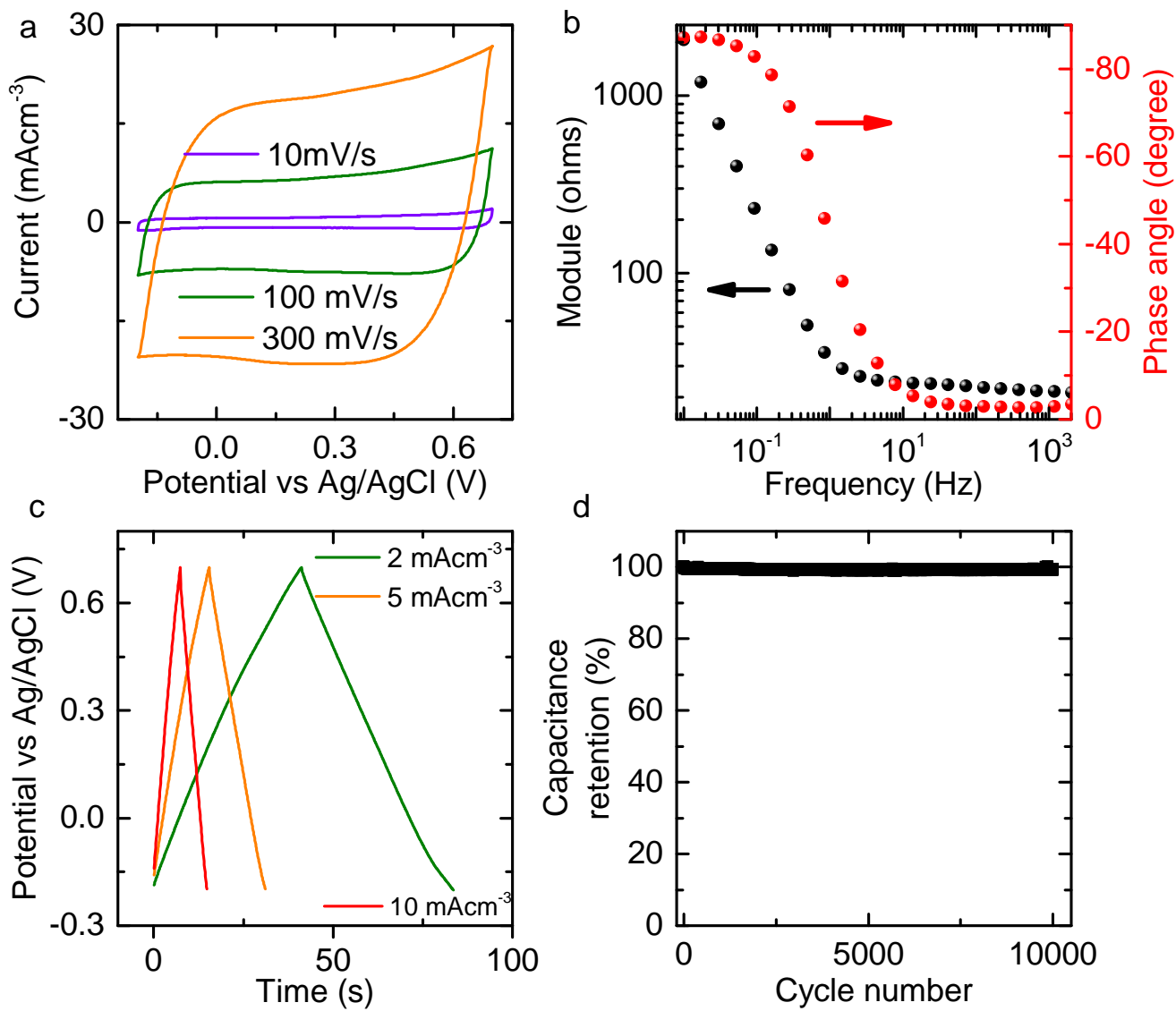

Figure 5. SEM and electrochemical characterization of the customized foams. (a) CV curves and (b) electrochemical impedance spectroscopy measurements in a $3 \mathrm{M} \mathrm{KCl}$ electrolyte of a $\mathrm{GF}$ grown at $950{ }^{\circ} \mathrm{C}$ using $\mathrm{Cu}(\mathrm{Mg}) 1 \mu \mathrm{m}$ powder. (c) Charge-discharge curves at different volumetric currents. (d) Endurance test of the same sample as in (c) showing nearly no loss after 10,000 cycles of charge-discharge experiments. 
shape shown in Fig. 4b. Charge-discharge measurements (Fig. 5c), carried out at different volumetric currents (current per unit volume), reveal a quasi-triangular shape of the potential-time curves without any visible potential saturation, which would be typically observed in presence of electrochemical reactions[19]. Thus, charge-discharge measurements indicate a characteristic fingerprint of an ideal capacitive interface of the GF electrodes[62]. Endurance tests are carried out over 10,000 cycles at a volumetric current of $4 \mathrm{~mA} \mathrm{~cm}^{-3}$, which results in a charge time of three seconds, typical for supercapacitor applications[11]. Such tests (Fig. 5d) reveal no significant degradation of the electrode performance with a total capacitance drop lower than $1 \%$.

\section{Conclusion}

In summary, we have demonstrated a process for the CVD growth of free-standing 3D single layer and few layer graphene foams with both high volumetric and specific capacitance for EDLC applications. To achieve this target, we designed and realized the metal template by sintering $\mathrm{Cu}$ and $\mathrm{Ni}$ metal powders of smaller particle size (average particle size smaller than $10 \mu \mathrm{m}$ ) at high temperatures, in contrast to commercial processes where larger particles $(>100 \mu \mathrm{m})$ or metal hydrides are used. In order to prevent agglomeration of particles, the powders were either not compressed or a blowing agent $\left(\mathrm{MgCO}_{3}\right)$ was used. Our proposed approach is versatile with GFs having pore sizes smaller than $1 \mu \mathrm{m}$, which can be tuned by changing both the metal particle size and the growth temperature. Moreover, our proposed approach has the possibility to tune on-demand the mass as well as the mechanical stability of the as-grown GFs by changing the catalyst template with consequent control on layer number. The high EASA of the fabricated foams, $10^{4}$ larger than the surface area of GFs prepared using commercial $\mathrm{Ni}$ or $\mathrm{Cu}$ templates, makes the proposed GFs very attractive electrodes for energy storage applications. We have tested our GFs prepared using $\mathrm{Cu}$ powder in EDLCs and a volumetric capacitance of $165 \mathrm{mF} \mathrm{cm}^{-3}$ was achieved which is almost five orders of magnitude higher than the one achieved with commercial $\mathrm{Ni}$ foams $\left(5 \mu \mathrm{F} \mathrm{cm} \mathrm{cm}^{-3}\right)$ grown under the same experimental conditions. In order to increase the specific capacitance, we have also demonstrated the growth of GFs on $\mathrm{Cu}$ templates consisting mostly of SLG, yielding a specific capacitance of $100 \mathrm{~F} \mathrm{~g}^{-1}$.

\section{Methods}

\section{Fabrication of GFs}

$\mathrm{Ni}$ (size $\mathrm{A}$ and size B) and $\mathrm{Cu}$ metal powders (Sigma Aldrich, see S.I. for particle size distribution) were used as metal substrate for the graphene growth. A Cu (size B) powder (Sigma Aldrich, particle size between 0.5 and $1.5 \mu \mathrm{m}$ ) was additionally mixed with $\mathrm{MgCO}_{3}$ powder and compressed (see also S.I.). Here, the compression was controlled by a fixed amount of powder used for the same volume. The powder was filled in a quartz combustion vessel $\left(15 \times 6 \times 4.5 \mathrm{~mm}^{3}\right)$ and inserted into a quartz tube (outer 
diameter $56 \mathrm{~mm}$ ) subsequently placed in a horizontal tube furnace (CarboLite HST 12/600). An annealing step with a flow of $400 \mathrm{sccm}$ argon and $100 \mathrm{sccm}$ hydrogen at high temperature for 45 minutes was used to connect the metal particles (thus forming the metal scaffold) as well as to remove metal oxides and contamination. Carbon feedstock was provided by a methane flow of $10 \mathrm{sccm}$ under a constant argon flow of $400 \mathrm{sccm}$ and a total pressure of $50 \mathrm{mbar}$ and $400 \mathrm{mbar}$ for the case of the growth on $\mathrm{Ni}$ and $\mathrm{Cu}$, respectively, which were the minimum pressures yielding closed graphene layers on the metal templates at all the investigated temperatures. The annealing process was carried out at the same temperature used for the growth. After 30 minutes, the $\mathrm{Ni}$ foam was cooled down in an argon atmosphere with a slow cooling rate of 10 to $40{ }^{\circ} \mathrm{C} \mathrm{min}^{-1}$ to enable the diffusion of carbon in the bulk to the surface of the metal template; the $\mathrm{Cu}$ foam, on the other hand, was immediately removed from the oven after the growth by extracting the quartz tube from the furnace resulting in a high cooling rate of $6{ }^{\circ} \mathrm{Cs}^{-1}$ to minimize the formation of defects[63]. After the growth process, the metal (either $\mathrm{Ni}$ or $\mathrm{Cu}$ ) and if necessary the filling powder was removed in a bath of $1 \mathrm{M} \mathrm{FeCl}_{3}$ and $2 \mathrm{M} \mathrm{HCl}$ for $\approx 6$ hours. The etchant can enter the metal template through defects in the graphene layer(s) such as vacancies and grain boundaries. The resulting GF was then cleaned in $\mathrm{HCl}(4 \mathrm{M})$ and finally in deionized (DI) water. The growth and transfer protocols of GFs grown on commercial Ni foam were identical to the ones performed for the GFs grown on the customized Ni templates. In order to prevent the collapse of the GFs, the GFs were freeze-dried at low pressure in liquid nitrogen for several hours.

\section{SEM Measurements and Pore Size Quantification}

SEM (Zeiss, SEMEvo, $5 \mathrm{kV}$ acceleration voltage) was used to characterize the structure of the GF and the metal foam. SEM images were also used to quantify the outer and inner pore sizes. Here, for each sample, all pore sizes were measured in nine different areas $\left(\approx 1000 \mathrm{\mu m}^{2}\right.$ each). The error bars reported in Fig. 4a correspond to the standard deviation of this measurement.

\section{TEM}

Small portions of the GF have been fragmented by ultrasonication for 5 minutes in isopropanol. The dispersions were drop-cast on Au-supported holey carbon grids for TEM characterization. Structural and compositional characterization of the materials has been carried out on FEI Tecnai F20 ST Transmission Electron Microscope, operated at $120 \mathrm{kV}$ of accelerating voltage to reduce the beam damage on the graphene flakes.

\section{Raman}

Raman measurements in Fig. 2a-d were carried out with a Renishaw 1000 at $532 \mathrm{~nm}$ excitation wavelength and a $100 \mathrm{X}$ objective, with an incident power of $\approx 1 \mathrm{~mW}$. The Raman maps in Fig. 2c-d, as well as the Raman maps shown in the S.I., were measured 
by WiTec using an excitation wavelength of $532 \mathrm{~nm}$ in a confocal system, which allows the spatial resolution down to $200 \mathrm{~nm}$. The growth temperature dependence of the $\mathrm{I}(2 \mathrm{D}) / \mathrm{I}(\mathrm{G})$ and the $\mathrm{I}(\mathrm{D}) / \mathrm{I}(\mathrm{G})$ in Fig 2e-f were recorded with an excitation wavelength of $514.5 \mathrm{~nm}$. Here, a $30 \times 30 \mathrm{\mu m}^{2}$ Raman map was measured with an integration time of 20 seconds and a step size of $5 \mu \mathrm{m}$. The D, G, and 2D peaks were fitted with Lorentzian functions.

\section{Electrochemical Measurements}

In order to investigate the electrochemical properties, the GFs were transferred to a glassy carbon $\left(10 \times 10 \mathrm{~mm}^{2}\right)$ substrate, which was placed on a Au/Ti layer evaporated on a glass slide. An aluminum wire was soldered to the gold layer. For the particular case of the GFs grown using the $\mathrm{Cu}(\mathrm{Mg}) 1 \mu \mathrm{m}$ templates, the GFs were transferred to a multilayer graphene sheet (MLGS), which was placed on a glass slide. The MLGS was grown on $\mathrm{Ni}$ foil $\left(25 \mu \mathrm{m}\right.$ thickness) by $\mathrm{CVD}$ at $900^{\circ} \mathrm{C}$ using the same procedure as for the growth of GFs on Ni templates and was contacted by an aluminum wire. In all cases, Scrintec 601 was used to insulate the wiring and to prevent the contact of the electrolyte $(3 \mathrm{M} \mathrm{KCl})$ with the substrate. All electrochemical measurements were recorded by Parstat (Princeton Applied Technologies) in a three electrode system. A platinum wire served as counter electrode and a $\mathrm{Ag} / \mathrm{AgCl}$ electrode was used as reference.

\section{Acknowledgements}

We thank Dr. Ute Schmidt of WITec (Wissenschaftliche Instrumente und Technologie $\mathrm{GmbH}$ ) for three-dimensional Raman measurements in a confocal configuration. This work has been partially supported by the German Research Foundation (DFG) in the framework of the Priority Program 1459 Graphene, the Nanosystems Initiative Munich (NIM), and the European Unions Horizon 2020 research and innovation programme under grant agreement No. 696656 GrapheneCore1.

\section{References}

[1] Zongping Chen, Wencai Ren, Libo Gao, Bilu Liu, Songfeng Pei, and Hui-Ming Cheng. Threedimensional flexible and conductive interconnected graphene networks grown by chemical vapour deposition. Nature materials, 10(6):424-428, 2011.

[2] K. S. Novoselov, A. K. Geim, S. V. Morozov, D. Jiang, Y. Zhang, S. V. Dubonos, I. V. Grigorieva, and A. A. Firsov. Electric field effect in atomically thin carbon films. Science (New York, N.Y.), 306(5696):666-669, 2004.

[3] K. S. Novoselov, V. I. Fal'ko, L. Colombo, P. R. Gellert, M. G. Schwab, and K. Kim. A roadmap for graphene. Nature, 490(7419):192-200, 2012.

[4] Andrea C. Ferrari, Francesco Bonaccorso, Vladimir Fal'ko, Konstantin S. Novoselov, Stephan Roche, Peter Bøggild, Stefano Borini, Koppens, Frank H. L., Vincenzo Palermo, Nicola Pugno, José A. Garrido, Roman Sordan, Alberto Bianco, Laura Ballerini, Maurizio Prato, Elefterios Lidorikis, Jani Kivioja, Claudio Marinelli, Tapani Ryhänen, Alberto Morpurgo, 
Jonathan N. Coleman, Valeria Nicolosi, Luigi Colombo, Albert Fert, Mar Garcia-Hernandez, Adrian Bachtold, Grégory F. Schneider, Francisco Guinea, Cees Dekker, Matteo Barbone, Zhipei Sun, Costas Galiotis, Alexander N. Grigorenko, Gerasimos Konstantatos, Andras Kis, Mikhail Katsnelson, Lieven Vandersypen, Annick Loiseau, Vittorio Morandi, Daniel Neumaier, Emanuele Treossi, Vittorio Pellegrini, Marco Polini, Alessandro Tredicucci, Gareth M. Williams, Byung Hee Hong, Jong-Hyun Ahn, Jong Min Kim, Herbert Zirath, van Wees, Bart J., van der Zant, Herre, Luigi Occhipinti, Andrea Di Matteo, Ian A. Kinloch, Thomas Seyller, Etienne Quesnel, Xinliang Feng, Ken Teo, Nalin Rupesinghe, Pertti Hakonen, Neil, Simon R. T., Quentin Tannock, Tomas Löfwander, and Jari Kinaret. Science and technology roadmap for graphene, related two-dimensional crystals, and hybrid systems. Nanoscale, 7(11):4598-4810, 2015.

[5] K. I. Bolotin, K. J. Sikes, Z. Jiang, M. Klima, G. Fudenberg, J. Hone, P. Kim, and H. L. Stormer. Ultrahigh electron mobility in suspended graphene. Solid State Communications, 146(9-10):351$355,2008$.

[6] Alexander A. Balandin, Suchismita Ghosh, Wenzhong Bao, Irene Calizo, Desalegne Teweldebrhan, Feng Miao, and Chun Ning Lau. Superior thermal conductivity of single-layer graphene. Nano letters, 8(3):902-907, 2008.

[7] Haiqun Chen, Marc B. Müller, Kerry J. Gilmore, Gordon G. Wallace, and Dan Li. Mechanically Strong, Electrically Conductive, and Biocompatible Graphene Paper. Advanced Materials, 20(18):3557-3561, 2008.

[8] Changgu Lee, Xiaoding Wei, Jeffrey W. Kysar, and James Hone. Measurement of the elastic properties and intrinsic strength of monolayer graphene. Science (New York, N.Y.), 321(5887):385-388, 2008.

[9] A. K. Geim. Graphene: status and prospects. Science (New York, N.Y.), 324(5934):1530-1534, 2009.

[10] Meryl D. Stoller, Sungjin Park, Yanwu Zhu, Jinho An, and Rodney S. Ruoff. Graphene-based ultracapacitors. Nano letters, 8(10):3498-3502, 2008.

[11] Patrice Simon and Yury Gogotsi. Materials for electrochemical capacitors. Nature materials, $7(11): 845-854,2008$.

[12] Yuyan Shao, Jun Wang, Hong Wu, Jun Liu, Ilhan A. Aksay, and Yuehe Lin. Graphene Based Electrochemical Sensors and Biosensors: A Review. Electroanalysis, 22(10):1027-1036, 2010.

[13] F. Bonaccorso, L. Colombo, G. Yu, M. Stoller, V. Tozzini, A. C. Ferrari, R. S. Ruoff, and V. Pellegrini. Graphene, related two-dimensional crystals, and hybrid systems for energy conversion and storage. Science, 347(6217):1246501, 2015.

[14] A. Peigney, Ch. Laurent, E. Flahaut, R. R. Bacsa, and A. Rousset. Specific surface area of carbon nanotubes and bundles of carbon nanotubes. Carbon, 39(4):507-514, 2001.

[15] Jean-Baptiste Donnet, Roop Chand Bansal, and Meng-Jiao Wang. Carbon black: Science and technology. Dekker, New York, 2nd ed., rev. and expanded edition, 1993.

[16] Y. Zhu, S. Murali, M. D. Stoller, K. J. Ganesh, W. Cai, P. J. Ferreira, A. Pirkle, R. M. Wallace, K. A. Cychosz, M. Thommes, D. Su, E. A. Stach, and R. S. Ruoff. Carbon-Based Supercapacitors Produced by Activation of Graphene. Science, 332(6037):1537-1541, 2011.

[17] K. K. Diao, Z. Xiao, and Y. Y. Zhao. Specific surface areas of porous $\mathrm{Cu}$ manufactured by Lost Carbonate Sintering: Measurements by quantitative stereology and cyclic voltammetry. Materials Chemistry and Physics, 2015.

[18] S. Trasatti and R. Parsons. Interphases in systems of conducting phases (Recommendations 1985). Pure and Applied Chemistry, 58(3), 1986.

[19] Allen J. Bard and Larry R. Faulkner. Electrochemical methods: Fundamentals and applications. Wiley, New York, 2nd ed edition, 2001.

[20] Daniel R. Dreyer, Sungjin Park, Christopher W. Bielawski, and Rodney S. Ruoff. The chemistry of graphene oxide. Chem. Soc. Rev., 39(1):228-240, 2010.

[21] Sasha Stankovich, Dmitriy A. Dikin, Richard D. Piner, Kevin A. Kohlhaas, Alfred Kleinhammes, Yuanyuan Jia, Yue Wu, SonBinh T. Nguyen, and Rodney S. Ruoff. Synthesis of graphene-based 
nanosheets via chemical reduction of exfoliated graphite oxide. Carbon, 45(7):1558-1565, 2007.

[22] Linh T. Le, Matthew H. Ervin, Hongwei Qiu, Brian E. Fuchs, and Woo Y. Lee. Graphene supercapacitor electrodes fabricated by inkjet printing and thermal reduction of graphene oxide. Electrochemistry Communications, 13(4):355-358, 2011.

[23] Kaixuan Sheng, Yiqing Sun, Chun Li, Wenjing Yuan, and Gaoquan Shi. Ultrahigh-rate supercapacitors based on eletrochemically reduced graphene oxide for ac line-filtering. Scientific reports, 2:247, 2012.

[24] Long Zhang, Fan Zhang, Xi Yang, Guankui Long, Yingpeng Wu, Tengfei Zhang, Kai Leng, Yi Huang, Yanfeng Ma, Ao Yu, and Yongsheng Chen. Porous 3D graphene-based bulk materials with exceptional high surface area and excellent conductivity for supercapacitors. Scientific Reports, 3, 2013.

[25] Cecilia Mattevi, Goki Eda, Stefano Agnoli, Steve Miller, K. Andre Mkhoyan, Ozgur Celik, Daniel Mastrogiovanni, Gaetano Granozzi, Eric Garfunkel, and Manish Chhowalla. Evolution of Electrical, Chemical, and Structural Properties of Transparent and Conducting Chemically Derived Graphene Thin Films. Advanced Functional Materials, 19(16):2577-2583, 2009.

[26] X. Li, W. Cai, J. An, S. Kim, J. Nah, D. Yang, R. Piner, A. Velamakanni, I. Jung, E. Tutuc, S. K. Banerjee, L. Colombo, and R. S. Ruoff. Large-Area Synthesis of High-Quality and Uniform Graphene Films on Copper Foils. Science, 324(5932):1312-1314, 2009.

[27] Y. Gogotsi and P. Simon. True Performance Metrics in Electrochemical Energy Storage. Science, 334(6058):917-918, 2011.

[28] Kyoungsoo Kim, Taekyoung Lee, Yonghyun Kwon, Yongbeom Seo, Jongchan Song, Jung Ki Park, Hyunsoo Lee, Jeong Young Park, Hyotcherl Ihee, Sung June Cho, and Ryong Ryoo. Lanthanumcatalysed synthesis of microporous 3D graphene-like carbons in a zeolite template. Nature, 535(7610):131-135, 2016.

[29] Byung Hyun Min, Dae Woo Kim, Kyoung Hwan Kim, Hyung Ouk Choi, Sung Woo Jang, and Hee-Tae Jung. Bulk scale growth of CVD graphene on Ni nanowire foams for a highly dense and elastic 3D conducting electrode. Carbon, 80:446-452, 2014.

[30] Lu Zhang, Derek DeArmond, Noe T. Alvarez, Daoli Zhao, Tingting Wang, Guangfeng Hou, Rachit Malik, William R. Heineman, and Vesselin Shanov. Beyond graphene foam, a new form of threedimensional graphene for supercapacitor electrodes. J. Mater. Chem. A, 4(5):1876-1886, 2016.

[31] Junwei Sha, Caitian Gao, Seoung-Ki Lee, Yilun Li, Naiqin Zhao, and James M. Tour. Preparation of Three-Dimensional Graphene Foams Using Powder Metallurgy Templates. ACS nano, 10(1):1411-1416, 2016.

[32] Weiwei Li, Song Gao, Liqiong Wu, Shengqiang Qiu, Yufen Guo, Xiumei Geng, Mingliang Chen, Shutian Liao, Chao Zhu, Youpin Gong, Mingsheng Long, Jianbao Xu, Xiangfei Wei, Mengtao Sun, and Liwei Liu. High-density three-dimension graphene macroscopic objects for highcapacity removal of heavy metal ions. Scientific reports, 3:2125, 2013.

[33] Yoshikazu Ito, Yoichi Tanabe, H.-J. Qiu, Katsuaki Sugawara, Satoshi Heguri, Ngoc Han Tu, Khuong Kim Huynh, Takeshi Fujita, Takashi Takahashi, Katsumi Tanigaki, and Mingwei Chen. High-Quality Three-Dimensional Nanoporous Graphene. Angewandte Chemie, 126(19):49224926, 2014.

[34] Jong-Chul Yoon, Jung-Soo Lee, Sun-I Kim, Kwang-Hyun Kim, and Ji-Hyun Jang. ThreeDimensional Graphene Nano-Networks with High Quality and Mass Production Capability via Precursor-Assisted Chemical Vapor Deposition. Scientific Reports, 3, 2013.

[35] Junshuang Zhou, Jie Lian, Li Hou, Junchuan Zhang, Huiyang Gou, Meirong Xia, Yufeng Zhao, Timothy A. Strobel, Lu Tao, and Faming Gao. Ultrahigh volumetric capacitance and cyclic stability of fluorine and nitrogen co-doped carbon microspheres. Nature communications, 6:8503, 2015.

[36] Michael Ghidiu, Maria R. Lukatskaya, Meng-Qiang Zhao, Yury Gogotsi, and Michel W. Barsoum. Conductive two-dimensional titanium carbide 'clay' with high volumetric capacitance. Nature, 516(7529):78-81, 2014. 
[37] François Béguin, Volker Presser, Andrea Balducci, and Elzbieta Frackowiak. Supercapacitors: Carbons and Electrolytes for Advanced Supercapacitors (Adv. Mater. 14/2014). Advanced Materials, 26(14):2283, 2014.

[38] Alfonso Reina, Xiaoting Jia, John Ho, Daniel Nezich, Hyungbin Son, Vladimir Bulovic, Mildred S. Dresselhaus, and Jing Kong. Large area, few-layer graphene films on arbitrary substrates by chemical vapor deposition. Nano letters, 9(1):30-35, 2009.

[39] Xuesong Li, Carl W. Magnuson, Archana Venugopal, Rudolf M. Tromp, James B. Hannon, Eric M. Vogel, Luigi Colombo, and Rodney S. Ruoff. Large-Area Graphene Single Crystals Grown by Low-Pressure Chemical Vapor Deposition of Methane on Copper. Journal of the American Chemical Society, 133(9):2816-2819, 2011.

[40] John Banhart. Manufacturing routes for metallic foams. JOM, 52(12):22-27, 2000.

[41] Margarete Seeger, Walter Otto, Wilhelm Flick, Friedrich Bickelhaupt, and Otto S. Akkerman. Magnesium Compounds. In Ullmann's Encyclopedia of Industrial Chemistry. Wiley-VCH Verlag GmbH \& Co. KGaA, Weinheim, Germany, 2000.

[42] George F. Schrader, Ahmad K. Elshennawy, and Lawrence E. Doyle. Manufacturing processes 83 materials. Society of Manufacturing Engineers, Dearborn, Mich., 4th ed edition, 2000.

[43] HoKwon Kim, Cecilia Mattevi, M. Reyes Calvo, Jenny C. Oberg, Luca Artiglia, Stefano Agnoli, Cyrus F. Hirjibehedin, Manish Chhowalla, and Eduardo Saiz. Activation energy paths for graphene nucleation and growth on $\mathrm{Cu}$. ACS nano, 6(4):3614-3623, 2012.

[44] Ivan Vlassiouk, Murari Regmi, Pasquale Fulvio, Sheng Dai, Panos Datskos, Gyula Eres, and Sergei Smirnov. Role of Hydrogen in Chemical Vapor Deposition Growth of Large Single-Crystal Graphene. ACS nano, 5(7):6069-6076, 2011.

[45] Xuesong Li, Yanwu Zhu, Weiwei Cai, Mark Borysiak, Boyang Han, David Chen, Richard D. Piner, Luigi Colombo, and Rodney S. Ruoff. Transfer of Large-Area Graphene Films for HighPerformance Transparent Conductive Electrodes. Nano Letters, 9(12):4359-4363, 2009.

[46] Xuesong Li, Weiwei Cai, Luigi Colombo, and Rodney S. Ruoff. Evolution of Graphene Growth on $\mathrm{Ni}$ and $\mathrm{Cu}$ by Carbon Isotope Labeling. Nano Letters, 9(12):4268-4272, 2009.

[47] Alexander E. Karu. Pyrolytic Formation of Highly Crystalline Graphite Films. Journal of Applied Physics, 37(5):2179, 1966.

[48] J. J. Lander, H. E. Kern, and A. L. Beach. Solubility and Diffusion Coefficient of Carbon in Nickel: Reaction Rates of Nickel-Carbon Alloys with Barium Oxide. Journal of Applied Physics, 23(12):1305, 1952.

[49] Stephan Hofmann, Philipp Braeuninger-Weimer, and Robert S. Weatherup. CVD-Enabled Graphene Manufacture and Technology. The journal of physical chemistry letters, 6(14):2714$2721,2015$.

[50] Maria Losurdo, Maria Michela Giangregorio, Pio Capezzuto, and Giovanni Bruno. Graphene CVD growth on copper and nickel: role of hydrogen in kinetics and structure. Physical chemistry chemical physics : PCCP, 13(46):20836-20843, 2011.

[51] Francesco Bonaccorso, Antonio Lombardo, Tawfique Hasan, Zhipei Sun, Luigi Colombo, and Andrea C. Ferrari. Production and processing of graphene and 2d crystals. Materials Today, 15(12):564-589, 2012.

[52] Qingkai Yu, Jie Lian, Sujitra Siriponglert, Hao Li, Yong P. Chen, and Shin-Shem Pei. Graphene segregated on Ni surfaces and transferred to insulators. Applied Physics Letters, 93(11):113103, 2008.

[53] Yufeng Hao, Lei Wang, Yuanyue Liu, Hua Chen, Xiaohan Wang, Cheng Tan, Shu Nie, Ji Won Suk, Tengfei Jiang, Tengfei Liang, Junfeng Xiao, Wenjing Ye, Cory R. Dean, Boris I. Yakobson, Kevin F. McCarty, Philip Kim, James Hone, Luigi Colombo, and Rodney S. Ruoff. Oxygenactivated growth and bandgap tunability of large single-crystal bilayer graphene. Nature nanotechnology, 11(5):426-431, 2016.

[54] G. A. López and E. J. Mittemeijer. The solubility of C in solid Cu. Scripta Materialia, 51(1):1-5, 2004. 
[55] Seung Jin Chae, Fethullah Güneş, Ki Kang Kim, Eun Sung Kim, Gang Hee Han, Soo Min Kim, Hyeon-Jin Shin, Seon-Mi Yoon, Jae-Young Choi, Min Ho Park, Cheol Woong Yang, Didier Pribat, and Young Hee Lee. Synthesis of Large-Area Graphene Layers on Poly-Nickel Substrate by Chemical Vapor Deposition: Wrinkle Formation. Advanced Materials, 21(22):2328-2333, 2009.

[56] A. C. Ferrari and J. Robertson. Interpretation of Raman spectra of disordered and amorphous carbon. Physical Review B, 61(20):14095-14107, 2000.

[57] Andrea C. Ferrari and Denis M. Basko. Raman spectroscopy as a versatile tool for studying the properties of graphene. Nature Nanotechnology, 8(4):235-246, 2013.

[58] A. C. Ferrari, J. C. Meyer, V. Scardaci, C. Casiraghi, M. Lazzeri, F. Mauri, S. Piscanec, D. Jiang, K. S. Novoselov, S. Roth, and A. K. Geim. Raman Spectrum of Graphene and Graphene Layers. Physical Review Letters, 97(18), 2006.

[59] Xuesong Li, Carl W. Magnuson, Archana Venugopal, Jinho An, Ji Won Suk, Boyang Han, Mark Borysiak, Weiwei Cai, Aruna Velamakanni, Yanwu Zhu, Lianfeng Fu, Eric M. Vogel, Edgar Voelkl, Luigi Colombo, and Rodney S. Ruoff. Graphene films with large domain size by a two-step chemical vapor deposition process. Nano letters, 10(11):4328-4334, 2010.

[60] M. Poot and van der Zant, H. S. J. Nanomechanical properties of few-layer graphene membranes. Applied Physics Letters, 92(6):063111, 2008.

[61] Hengxing Ji, Xin Zhao, Zhenhua Qiao, Jeil Jung, Yanwu Zhu, Yalin Lu, Li Li Zhang, Allan H. MacDonald, and Rodney S. Ruoff. Capacitance of carbon-based electrical double-layer capacitors. Nature communications, 5:3317, 2014.

[62] Mark E. Orazem and Bernard Tribollet. Electrochemical Impedance Spectroscopy. John Wiley \& Sons, Inc, Hoboken, NJ, USA, 2008.

[63] Dong Soo Choi, Keun Soo Kim, Hyeongkeun Kim, Yena Kim, TaeYoung Kim, Se-hyun Rhy, Cheol-Min Yang, Dae Ho Yoon, and Woo Seok Yang. Effect of cooling condition on chemical vapor deposition synthesis of graphene on copper catalyst. ACS applied materials $\mathcal{E}$ interfaces, 6(22):19574-19578, 2014. 